\title{
Analysis of quality HDMI connector component welding in Mother Board Laptop
}

\author{
Antônio da Silva Vieira ${ }^{1}$, Robson Marques Costa ${ }^{1}$, Cristiano Mourão Fonseca ${ }^{1}$, Carlos Alberto de Oliveira Freitas ${ }^{2}$, Roberto \\ Tetsuo Fujiyama ${ }^{1}$ \\ ${ }^{1}$ Programa de Pós Graduação em Engenharia Industrial-PPGEI/Universidade Federal do Pará-UFPA. Rua Augusto Correa 01 \\ Guamá. Belém - Pará - Brasil. \\ ${ }^{2}$ Programa de Pós Graduação em Engenharia Elétrica -PPGEE/Universidade Federal do Pará-UFPA. Rua Augusto Correa 01 \\ Guamá. Belém - Pará - Brasil. \\ Email:'1adasilvavieira@gmail.com, ${ }^{1}$ robson_m_costa@hotmail.com ${ }^{2}$,cristianomourao@yahoo.com,br, caofreitas@yahoo.com.br, \\ fujiyama@ufpa.br.
}

\begin{abstract}
The paper aims to assess the quality of HDMI connector component of welding in the welding process laptop printed circuit board meeting the eligibility criteria related to the filling of the solder barrel to pin through-hole components terminals as specified in international standard IPC-A-610D. For the development of the stages of research, it adopted the PDCA method, used for troubles hooting and application of continuous improvement, commonly used by different companies in the industrial segment worldwide. The results presented through analysis by X-RAY and metallographic test cross section, showed that the quality of HDMI welding through the automatic welding process by remelting meets the technical specifications of the standard of acceptability. Thus, left to perform the welding of this type of component in the manual process, passing it to weld using SMT technology.
\end{abstract}

Keywords: Quality, Welding Remelting, HDMI, IPC-A-610D and PCI.

\section{Análise da qualidade da soldagem do componente conector HDMI Mother Board Laptop}

\section{RESUMO}

O objetivo do artigo é avaliar a qualidade da soldagem do componente conector HDMI no processo de soldagem de Placas de Circuito Impresso de Laptop atendendo os critérios de aceitabilidade relacionados ao enchimento do barril de solda para terminais de componentes Pin Through-Hole conforme especificado na norma internacional IPC-A-610D. Para o desenvolvimento das etapas da pesquisa, adotou-se o método PDCA, utilizada para resolução de problemas e aplicação da melhoria contínua, comumente usada por diversas empresas do seguimento industrial em todo o mundo. Os resultados apresentados através de análise por raios-X e ensaio metalográfico de cross section, demostraram que a qualidade da soldagem do HDMI através do processo automático de soldagem por refusão atende ás especificações técnicas de aceitabilidade da norma. Deste modo, deixou de realizar a soldagem deste tipo de componente pelo processo manual, passando-o para a soldagem utilizando a Tecnologia SMT.

Palavras - chave: Qualidade, Soldagem por refusão, HDMI, IPC-A-610D e PCI.

\section{INTRODUÇÃO}

As transformações recorrentes nos processos de manufatura de produtos de informática nos últimos anos, têm se mostrado cada vez mais dinâmicas, tornando imprescindível o aperfeiçoamento continuo por parte das indústrias deste segmento, as quais adotam diferentes metodologias e técnicas de abordagens através da implantação de programas de melhorias nos processos de soldagem de Placas de Circuito Impresso (PCI).
Dentro deste contexto, destaca-se o componente conector High Definition Multimedia Interface (HDMI), que utiliza um sistema de conexão de alta tecnologia capaz de transmitir áudio e vídeo através de um único cabo, e que é de grande utilidade pelos usuários para transmissão de dados em alta definição. Anterior a esta tecnologia, cada tipo de transmissão possuía cabos e entradas exclusivas nos aparelhos. É atualmente o melhor e mais usado para transmissão de áudio e vídeo digital. Permite, por

ITEGAM - JETIA Vol. 01, Nº 02. Junho de 2015. Manaus - Amazonas, Brasil. ISSN 2447-0228 (ONLINE). 
exemplo, exibir imagens de alta definição de um notebook para um televisor LED ou projetor de imagens.

No processo de soldagem deste componente em um modelo de laptop, foi identificado um problema de ordem técnica, ou seja, o processo de soldagem não atendia os critérios internacionais de soldabilidade relacionado ao enchimento do barril de solda para os terminais do componente Pin ThroughHole (PTH). Tal ítem tecnológico é amplamente demandando no mercado nacional e internacional, não devendo apresentar anormalidade que por ventura acarrete insatisfação por parte do consumidor final. No entanto, no processo fabril de laptops, ainda ocorrem muitos problemas relacionados á soldagem de componentes eletrônicos; desta forma, a qualidade e a confiabilidade do produto podem estar diretamente comprometidas.

Portanto, com base neste ambiente dinâmico, propõe-se o desenvolvimento de um estudo no processo de soldagem do HDMI em placas de laptop em uma empresa do Polo Industrial de Manaus (PIM), de tal forma que viabilize uma solução técnica para o problema de soldabilidade. Para realização da pesquisa, estudou-se o processo de soldagem do componente HDMI em três modelos de laptop abrangendo os processos de soldagem automática por refusão através da Tecnologia de Montagem em Superfície (SMT) e do processo de soldagem manual.

A maioria das ferramentas, abordagens e técnicas utilizadas nos processos de fabricação para solucionar problemas, já é uma realidade nas grandes empresas em todo o mundo por serem de fácil compreensão. Um exemplo é o método Plan, Do, Check e Act (PDCA), ou círculo de Deming, cujo objetivo é a melhoria da qualidade dos processos de fabricação e das atividades que respondam às necessidades da organização, sendo este um esforço contínuo e permanente para atingir melhorias mensuráveis na eficiência, eficácia, desempenho, responsabilidade, resultados e outros indicadores de qualidade [1].

O grande desafio das montadoras de PCI tem sido de garantir a qualidade de seus produtos atendendo os critérios internacionais de aceitabilidade da norma IPC-A-610D, cuja mesma, define padrões de aceitabilidade de qualidade, bem como, assegurar que produtos de alta qualidade sejam oferecidos ao cliente final. É um padrão extensamente utilizado pela indústria que define a aceitabilidade de vários tipos de defeitos de soldagem [2].

Deste modo, para atender esta problemática e buscar soluções para resolver um problema de ordem técnica, tal pesquisa torna-se, relevante para a empresa em estudo e para as demais empresas do segmento de informática, pois alerta para o problema de insuficiência no enchimento do barril de solda em componentes com tecnologia PTH, aplicável para conectores HDMI e, de fácil extensão para outros tipos de conectores utilizados em: laptop, desktop, tablet, smartphone, entre outros produtos de informática.

\section{REVISÃO BIBLIOGRÁFICA}

\subsection{Processo de soldagem por refusão}

Após a montagem dos componentes, a PCI é transportada "in line" para um forno de refusão onde se inicia o processo de soldagem. Este processo consiste no aquecimento dos componentes, da placa e no derretimento da pasta de solda até formar uma junta de solda [3]. A soldagem é realizada através de um forno conhecido com forno de refusão, que é comumente usado para sistemas de convecção forçada para médias temperaturas [4]. O forno de refusão aquece a PCI a uma temperatura apropriada, por um período de tempo e taxa de aquecimento adequado através de zonas de aquecimento [5].

Para um processo ideal de soldagem por refusão, é elaborado um perfil térmico em que se definem os parâmetros de temperatura para cada zona do forno. Este perfil é uma curva da temperatura, uma relação do aquecimento da placa em relação ao tempo e controle dos efeitos do aquecimento. As temperaturas das zonas do forno devem ser homogêneas de modo a assegurar uma boa formação da junta de solda entre a ilha de solda da placa e o terminal do componente durante o processo de soldagem.

\subsection{Processo de Soldagem Manual de Componentes}

A soldagem manual é uma das tarefas mais difíceis no processo de fabricação de produtos de informática e eletroeletrônicos. Neste processo é possível surgir problemas, como: curto de solda, insuficiência de solda, entre outros. Para a operação de soldagem manual, utiliza-se um ferro de solda que é usado para derreter a solda que vem em forma de fio. O ferro de solda, por mais limpo que ele esteja, transmitirá contaminação para a PCI, adicionando impurezas ao estanho aplicado nos terminais do componente durante o processo de soldagem.

\subsection{Método PDCA}

Em uma planta de processo, os resultados reais de uma ação são em comparação com uma meta ou um ponto do processo a ser resolvido. A diferença entre o planejado e o executado é então medidas corretivas a serem adotadas e para verificar a grande disparidade existente. A natureza repetida ou de kaizen segue esta definição usual de controle e é representado pelo PDCA [6][7]. É também conhecido como o circulo W. E. Deming.

Plan- identificar o problema a ser examinado, formular um problema específico, definir claramente o problema, estabelecer metas mensuráveis e atingíveis, identificar as partes interessadas e desenvolver uma comunicação com todos os envolvidos através de canais de comunicação para obter a aprovação. Doestabelecer critérios de sucesso experimental, para testar hipóteses, obter a aprovação das partes interessadas e apoio para a solução escolhida e implemetar solução. Check- reunir e analisar dados, validar ou não a hipótese. Act - Identificar mudanças sistêmicas e necessidades de formação para a aplicação, plano de monitoramento contínuo da solução e procurar outras oportunidades de melhoria [8]. A figura 1 mostra o ciclo PDCA. 


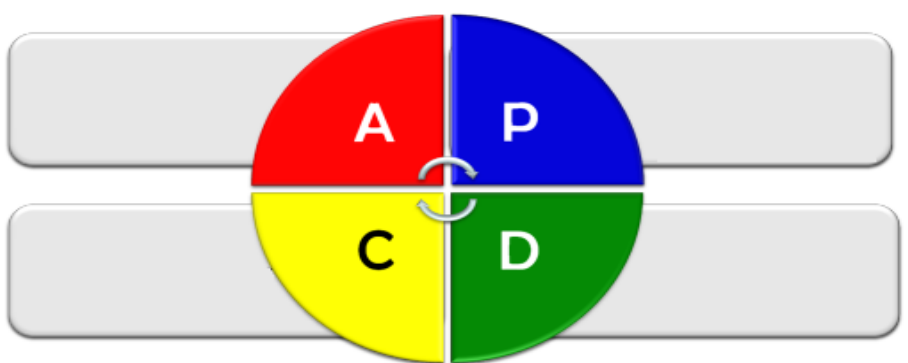

Figura 1. Ciclo PDCA.

\subsection{Tecnologia Pin in Paste (PIP)}

O processo PIP consiste na aplicação da pasta de solda nos furos da PCI onde são inseridos os terminais passantes dos componentes PTH. O processo é realizado na mesma etapa de impressão da pasta de solda nas ilhas dos componentes SMDs, que por sua vez, são montados através das máquinas Pick \& Place, e por fim, são soldados em uma única passagem pelo forno de refusão. Se a disposição do componente PTH não for própria para o processo automático de montagem, ele poderá ser inserido manualmente na PCI [9]. É a substituição dos processos de soldagem manual pelo processo de soldagem automática, denominado soldagem por refusão. Ainda o mesmo autor descreve que para este processo, devem ser consideradas algumas particularidades como: a espessura do stencil, o design para abertura no stencil, as dimensões do furo na PCI para os terminias e as dimensões do terminal do componente.

A figura 2 mostra o princípio da tecnologia Pin in Paste para um componente do tipo PTH.
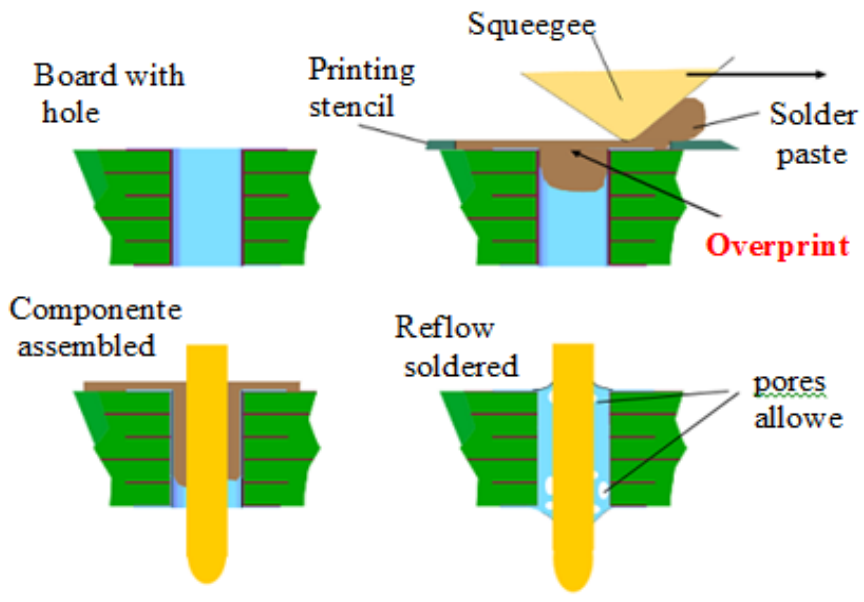

Figura 2. Princípio da tecnologia Pin in Paste [11].

Para o enchimento do barril de solda de um componente do tipo PTH, adotam-se alguns critérios de aceitabilidade estabelecidos na norma internacional IPC-A-610D para as classes 1,2 e 3 [11]. O enchimento mínimo aceitável é de 75\%, considerando um total máximo de $25 \%$ de depressão da solda em cima ou embaixo do barril, isso significa que parte da solda depositada na ilha de solda da PCI não escorre para o furo [12]. A Tabela 1 apresenta estes critérios.

Tabela 1. Critérios de Aceitabilidade de solda para as Classes de PCI's. [12].

\begin{tabular}{|l|c|c|c|}
\hline \multicolumn{1}{|c|}{ Critérios } & Classe 1 & Classe 2 & Classe 3 \\
\hline Molhagem da circunferência no lado primário & Não especificado & $180^{\circ}$ & $270^{\circ}$ \\
\hline Enchimento vertical de solda & Não especificado & $75^{\circ}$ & $75^{\circ}$ \\
\hline $\begin{array}{l}\text { Filete da circunferência e molhagem no lado secundário } \\
\text { (lado da fonte de solda) do terminal e poço }\end{array}$ & $270^{\circ}$ & $270^{\circ}$ & $270^{\circ}$ \\
\hline $\begin{array}{l}\text { Porcentagem de área da ilha coberta com solda molhada no } \\
\text { lado primário (lado de destino) }\end{array}$ & 0 & 0 & 0 \\
\hline $\begin{array}{l}\text { Porcentagem de área da ilha coberta com solda molhada no } \\
\text { lado secundário (lado da fonte de solda) }\end{array}$ & $75 \%$ & $75 \%$ & $75 \%$ \\
\hline
\end{tabular}

Visto que $75 \%$ de solda não represente um enchimento completo do barril (ideal é 100\%), no entanto, comprova-se tecnicamente que o componente apresentará uma boa conexão da junta de solda suficiente para garantir uma boa resistência aos esforções mecânicos durante a utilização do componente no aparelho. A Figura 3 mostra o enchimento mínimo aceitável pela IPC-A-610D para o barril de solda de um componente PTH.

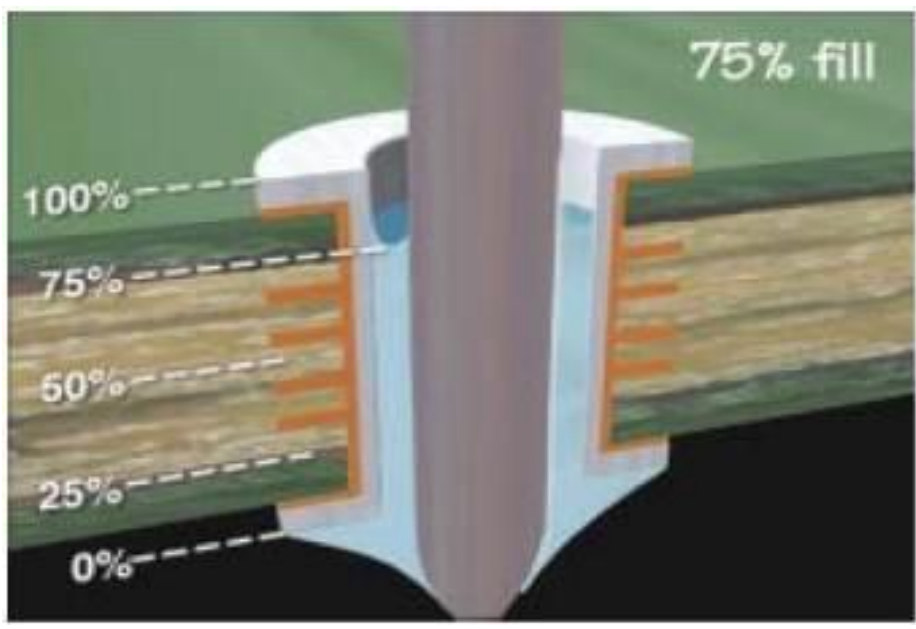

Figura 3. Enchimento de $75 \%$ de solda no barril do componente PTH aceitável pela norma IPC-A-610D [12].

\section{5 Inspeção por Raio-X}

Componentes mais complexos de soldagem têm possibilidades maiores de apresentar defeitos. Como uma técnica não destrutiva, a inspeção por Raio-X pode ser usada sem a necessidade de sacrificar o produto (aparelho), e ao mesmo tempo, ser capaz de identificar as falhas ocultas[13]. De acordo com a IPC-A-610D, alguns defeitos podem ser visualizados através da máquina de Raio-X, como: solder bridging e void. É considerado defeito se a área de void corresponder mais que $25 \%$ da área total da imagem.

A inspeção através do equipamento de Raio-X 2D ou 3D, é possível avaliar a confiabilidade dos processos de soldagem por refusão, soldagem por onda de solda e soldagem manual; o que o torna este equipamento extremamente importante e indispensável para o processo de análise da qualidade da soldagem de PCI. 


\section{6 Análise Metalográfica de Cross Section}

Análise microseccional ou análise de cross section (corte transversal) continua sendo o meio amplamente mais aceito e eficaz na avaliação da caracterização microestrutural de um material. Este ensaio é utilizado para confirmar a presença de falha da junta de solda [14]. O ensaio de cross section é um dos mais úteis métodos de análise metalográfica e, que pode revelar muito mais informações sobre a qualidade e composição de uma junta de solda do que uma simples análise de superfície. As áreas de investigação para análise metalográfica na eletrônica, incluem: rachaduras (cracks), vazios (voids), microestrutura, dimensões, defeitos de chapeamento, molhagem da solda (wetting), corrosão, PCI, composição, solda e geometria [15].

\section{METODOLOGIA DA PESQUISA}

O presente artigo buscou de forma prática, "in loco", avaliar o problema da pesquisa em questão, aplicando o método PDCA e com o auxílio do Diagrama de Causas e Efeito (Ishikawa). Tendo como base esses conceitos, avaliou-se a possibilidade de utilizar a ferramenta $5 \mathrm{~W} 2 \mathrm{H}$ para coletar as informações, organizar as tarefas apontadas como relevantes, elaborar o plano de ações corretivas e propor melhorias. Utilizar o diagrama de Ishikawa para analisar as possíveis causas do problema, bem como, identificar a causa raiz; porque trata-se de uma necessidade imediata do processo produtivo e, sobretudo, de interesse real da empresa em estudo.

\section{MATERIAIS E APLICAÇÃO.}

A pesquisa foi desenvolvida durante 90 dias no processo fabricação de mother board para laptops, abrangendo o processo de soldagem automática por refusão e processo de soldagem manual do componente conector HDMI. O estudo foi aplicado em três modelos de laptop. Para uma fácil compreensão, serão chamados de: Mother Board 1, para o primeiro modelo de laptop, Mother Board 2, para o segundo modelo de laptop e Mother Board 3, para o terceiro modelo de laptop ou MB1, MB2/MB3. A Figura 4 mostra os modelos dos laptops.
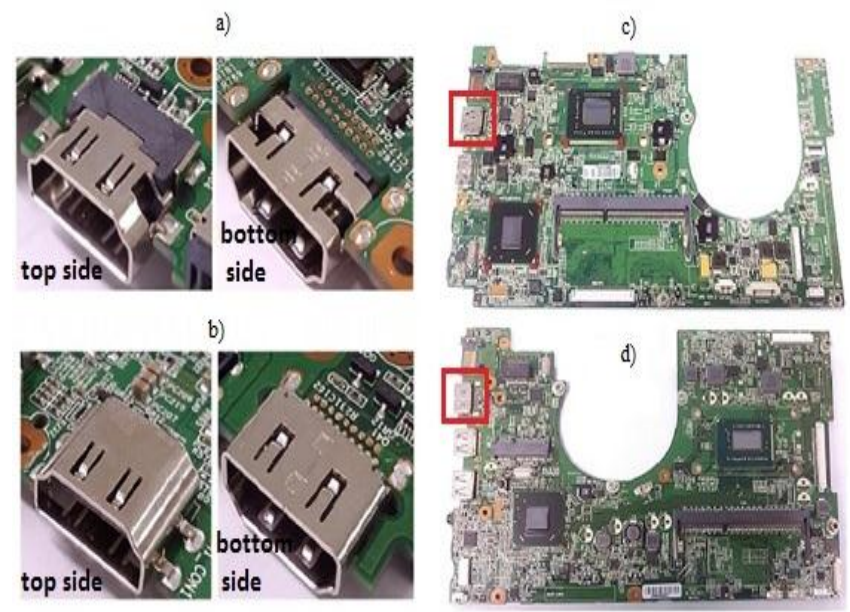

Figura 4. a) HDMI soldado na PCI modelo MB1(c); b) HDMI soldado na PCI modelos MB2/MB3 (d).

\subsection{Aplicação do ciclo PDCA na pesquisa}

Para o desenvolvimento do ciclo PDCA, a pesquisa foi realizada em várias etapas:

\subsubsection{Identificação do Problema da Pesquisa}

A empresa em estudo segue a metodologia amostral de teste de cliente final chamada Out of Box (OOB) com definição da amostra baseada na NBR 5426. Esta Norma estabelece planos de amostragem e procedimentos para inspeção por atributos. Em uma das amostras OOB dos modelos MB1, MB2 e MB3, foi evidenciado um problema de "Sem video no Monitor HDMI" durante o teste funcional do aparelho. A causa deste problema estava relacionada a um "curto de solda no componente HDMI". $\mathrm{O}$ fato importante evidenciado na amostragem OOB, foi que durante a investigação da causa do problema, detectou-se através de avaliação pelo equipamento de Raio-X que existia uma nãoconformidade no enchimento do barril de solda do HDMI, ou seja, não atendia a especificação da Norma IPC-A-610D.

\subsubsection{Observação e Análise do Problema}

Para efeito de simplificação, chamaremos de cenário 1, a situação inicial do processo de soldagem do HDMI. Os procedimentos padrão para a realização da operação de soldagem são definidos por uma empresa chinesa, fornecedora de insumos. A soldagem era realizada manual pelos operadores de solda, utilizando: um dispositivo para soldagem, uma estação de solda em fio.

A partir da evidência do problema durante a avaliação na amostragem, foi realizada uma analise mais profunda da qualidade da soldagem através do equipamento de Raio-X, em seguida, um ensaio metalográfico de cross section, onde foi possível detectar um problema de ordem técnica de insuficiência de solda no enchimento do barril dos terminais do componente. A Figura 5 mostra o resultado da análise por raio-x para os modelos MB1, MB2/MB3. a)

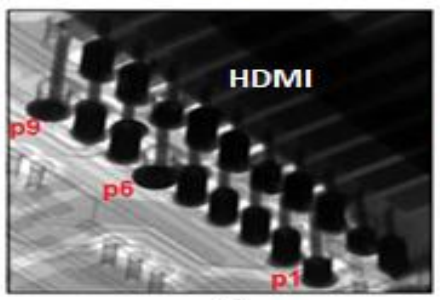

c)

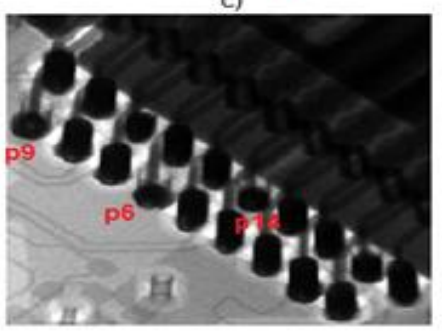

b)

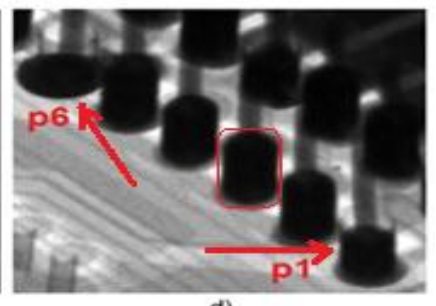

d)

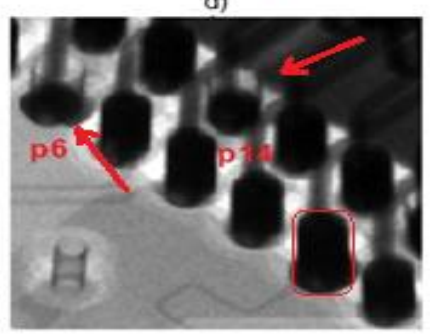

Figura 5. Raio-X do conector HDMI apresentando insuficiência de solda no barril. a) e b) modelo laptop MB1; c) e d) modelos laptop $\mathrm{MB} 2 / \mathrm{MB} 3$. 
Conforme mostrado na Figura 5, o resultado da análise de raio $\mathrm{X}$, após HDMI ter sido soldado no processo manual (cenário1), apresentou insuficiência de solda no barril dos terminais (p1) e (p6) no modelo MB1, e (p6) e (p14) nos modelos MB2/MB3, ou seja, houve enchimento de solda $<75 \%$, portanto, não atende o critério de aceitabilidade pela IPC-A610D.

O ensaio metalográfico de cross section foi realizado somente no modelo laptop MB1, porque o package do HDMI usado nos três modelos era muito semelhante, conforme mostrado na figura $4, \log$, não houve necessidade em realizar o ensaio metalográfico para os modelos MB2/MB3. Os resultados também evidenciaram o problema de ordem técnica de insuficiencia de solda, como mostra a figura 6.
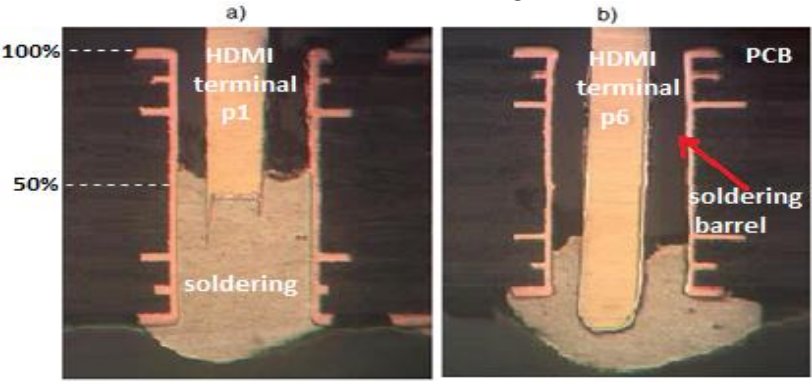

Figura 6. Cross section do conector HDMI modelo laptop MB1 apresentando insuficiência de solda no barril. a) terminal (p1) e b) terminal (p6). (50x).

Conforme mostrado na Figura 6, o resultado do ensaio metalográfico de cross section do HDMI após soldado no processo manual (cenário1), apresentou insuficiência de solda no barril dos terminais (p1) e (p6), ou seja, o enchimento do barril ficou $<75 \%$, portanto, fora da aceitabilidade da IPC-A-610D.

Diante do cenário apresentado nas figuras 5 e 6 , foi elaborado um Diagrama de Causa e Efeito, também conhecido mundialmente como diagrama de (Ishikawa) para analisar e avaliar as possíveis causas do problema. A Figura 7 mostra o Diagrama em questão.

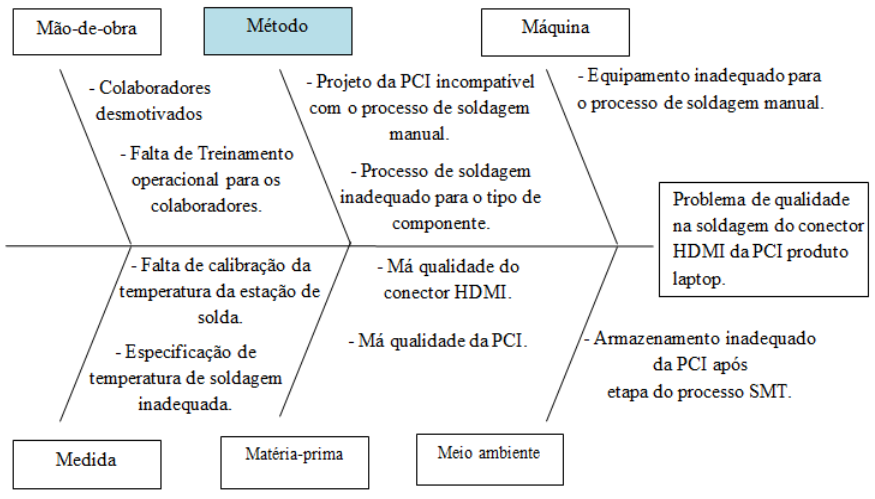

Figura 7. Diagrama de Causas e Efeito.

A guia método foi apontada como a causa raiz do problema, pelas seguintes razões: 1 - Avaliando o histórico de outros modelos de laptop, constatou-se que a soldagem do HDMI era realizada pelo processo automática utilizando a tecnologia Pin in Paste, e não existia histórico de problema nestes modelos. 2 - De acordo as informações técnicas do componente, o mesmo apresentava especificações para o processo de soldagem tanto pelo método manual quanto pelo método automático (por refusão). No entanto, na prática, o método de soldagem manual apresentou problemas de ordem técnica, conforme mostrado no cenário 1 . As demais possíveis causas apresentadas no diagrama foram avaliadas e concluiu-se que estavam de acordo para o processo de soldagem manual do componente em questão.

\subsubsection{Elaboração do Plano de Ação}

Após detectado a causa raiz do problema da pesquisa, foi elaborado um plano de ação para implementar a melhoria utilizando a ferramenta de qualidade $5 \mathrm{~W} 2 \mathrm{H}$ apresentado na tabela 2.

Tabela 2. 5W2H.

\begin{tabular}{|c|c|c|c|c|c|c|}
\hline (What) & (Why) & (How) & (When) & (Where) & (Who) & (How Much) \\
\hline $\begin{array}{c}\text { Transferir o } \\
\text { componente do } \\
\text { manual para o } \\
\text { processo } \\
\text { automático. }\end{array}$ & $\begin{array}{l}\text { Viabilizar a } \\
\text { soldagem } \\
\text { através das } \\
\text { máquinas } \\
\text { SMT. }\end{array}$ & $\begin{array}{c}\text { Incluir o } \\
\text { componente } \\
\text { no programa } \\
\text { das } \\
\text { máquinas. }\end{array}$ & $\begin{array}{l}\text { A partir de } \\
\text { Outubro } \\
\text { de } 2014\end{array}$ & $\begin{array}{c}\text { Na fase SMT } \\
\text { do processo } \\
\text { de fabricação } \\
\text { de laptop }\end{array}$ & $\begin{array}{l}\text { Antonio, Robson } \\
\text { e equipe de } \\
\text { programação de } \\
\text { máquinas SMT. }\end{array}$ & $\begin{array}{c}\text { Zero, pois } \\
\text { utilizou-se } \\
\text { mão-de-obra } \\
\text { intema. }\end{array}$ \\
\hline $\begin{array}{c}\text { Confecção de } \\
\text { cariers para } \\
\text { atender a nova } \\
\text { fase de montagem } \\
\text { SMT }\end{array}$ & $\begin{array}{l}\text { Viabilizar a } \\
\text { soldagem } \\
\text { através de } \\
\text { máquinas } \\
\text { SMT. }\end{array}$ & $\begin{array}{c}\text { Desenvolver } \\
\text { carriers } \\
\text { junto ao } \\
\text { fomecedor } \\
\text { extermo } \\
\text { especializado. }\end{array}$ & $\begin{array}{c}\text { A partir de } \\
\text { Outubro } \\
\text { de } 2014\end{array}$ & $\begin{array}{c}\text { Na fase SMT } \\
\text { do processo } \\
\text { de fabricação } \\
\text { de laptop }\end{array}$ & $\begin{array}{l}\text { Antonio, Robson } \\
\text { e equipe de } \\
\text { programação de } \\
\text { máquinas SMT. }\end{array}$ & $\begin{array}{c}\text { USD } \\
16.666,66\end{array}$ \\
\hline $\begin{array}{c}\text { Confecção de } \\
\text { novos stencils } \\
\text { para linha SMT } \\
\text { reconfigurada }\end{array}$ & $\begin{array}{l}\text { Viabilizar a } \\
\text { soldagem } \\
\text { através de } \\
\text { máquinas } \\
\text { SMT. }\end{array}$ & $\begin{array}{l}\text { Desenvolver } \\
\text { carriers } \\
\text { junto ao } \\
\text { formecedor } \\
\text { externo } \\
\text { especializado }\end{array}$ & $\begin{array}{c}\text { A partir de } \\
\text { Outubro } \\
\text { de } 2014\end{array}$ & $\begin{array}{c}\text { Na fase SMT } \\
\text { do processo } \\
\text { de fabricação } \\
\text { de laptop }\end{array}$ & $\begin{array}{l}\text { Antonio, Robson } \\
\text { e equipe de } \\
\text { programação de } \\
\text { máquinas SMT. }\end{array}$ & USD 916,66 \\
\hline
\end{tabular}

\section{RESULTADOS}

\subsection{Etapa CHECK (Verificar a eficácia da ação)}

As ações definidas no plano de ação foram implantadas, portanto, para esta segunda situação do processo de soldagem chamaremos, para efeito de simplificação, de cenário 2.

Analisando novamente, através do raio-X, o preenchimento do barril de solda dos terminais do HDMI, agora, pelo processo de soldagem automática, foi detectado conformidade da solda , ou seja, atendendo a norma IPC-A-610D. A Figura 8 mostra o resultado da análise para os modelos MB1, MB2/MB3.
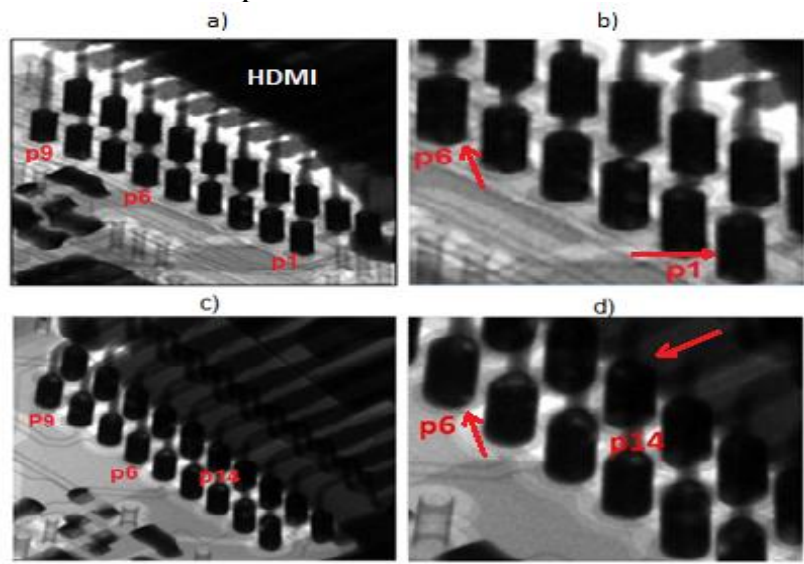

Figura 8. Raio-X do conector HDMI apresentando conformidade de solda no barril. a) e b) modelo laptop MB1; c) e d) modelos laptop MB2/MB3. 
Antônio da Silva Vieira et al./ ITEGAM-JETIA Vol.01, Nº 02, pp.10-16. Junho, 2015.

Conforme mostrado na Figura 8, o resultado da análise de Raio X, após HDMI ter sido soldado no processo automático (cenário2), apresentou enchimento de solda no barril dos terminais (p1) e (p6) no modelo MB1, e (p6) e (p14) nos modelos MB2/MB3, > 75\%, portanto, atendendo a IPC-A610D.

O ensaio metalográfico de cross section foi realizado, novamente, somente no modelo laptop MB1, pelas razões mencionadas anteriormente. Os resultados também evidenciaram enchimento de solda no barril dos terminais (p1) e (p6) > 75\% conforme mostrado na figura 9.
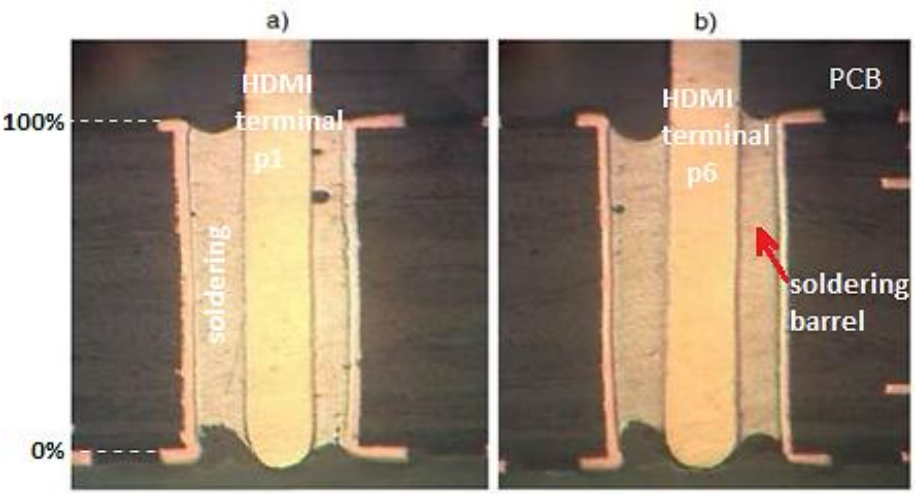

Figura 9. Cross section do conector HDMI modelo laptop MB1 apresentando conformidade de solda no barril. a) terminal (p1) e b) terminal (p6). (50x).

Conforme mostrado na Figura 9, o resultado do ensaio metalográfico de cross section do HDMI após soldado no processo automático (cenário2), ou seja, o enchimento do barril ficou $>75 \%$, portanto tecnicamente, dentro da aceitabilidade da IPC-A-610D.

\subsection{Etapa ACTION (Padronização do Processo)}

A padronização da melhoria do processo de soldagem é acompanhada através do documento "Relatório de Defeito", sendo monitorado diariamente. A melhoria no processo de soldagem do HDMI, se estendeu para os três modelos de laptop. Com base nos resultados apresentados na etapa "Check", é possível afirmar que a melhoria realizada no processo de soldagem do conector HDMI encontrou resultados conformes quanto aos critérios de aceitabilidade de soldabilidade relacionado ao enchimento do barril de solda dos terminais do componente HDMI. A tabela 3 mostra um resumo dos resultados antes e após o processo de melhoria.

\section{CONCLUSÃO}

Verificou-se, pelos resultados da pesquisa, que a soldagem do Conector HDMI nos modelos MB1, MB2/MB3 pelo processo de soldagem automática por refusão (SMT) e uso da tecnologia Pin in Paste, se mostrou eficaz, melhorando assim, a qualidade da solda dos terminais do componente HDMI, bem como, o atendimento da norma internacional IPC-A-610D sessão Supported Holes - Solder - Verticall Fill.

Identificou-se através das análises por Raio-X e de Ensaio Metalográfico de Cross Section o problema da pesquisa de ordem técnica, sendo que após a melhoria implantada no processo de soldagem, constatou-se por meio das mesmas análises, sua eficácia.

Orientado pelo método PDCA, o mesmo, mostrou-se eficiente tanto para a identificação da causa raiz do problema da pesquisa quanto para o desenvolvimento das etapas do estudo de caso.

Padronizou-se o processo de soldagem para este tipo de componente Conector HDMI em placas de laptop para os projetos futuros da empresa em estudo.

A pesquisa tornou-se relevante não somente para a empresa em estudo, mas, para as demais empresas do mesmo segmento de informática e eletroeletrônico, pois alerta para o problema de não atendimento a norma IPC-A-610D, quanto á insuficiente do enchimento do barril de solda em componentes PTH, bem como, apresenta uma solução técnica para situações desta natureza em conectores HDMI, e de fácil extensão para os demais conectores do mesmo tipo utilizados em laptops.

\section{REFERÊNCIAS BIBLIOGRÁFICAS}

[1] SOKOVIC, M, D Pavletic, K. Kern Pipan Quality Improvement Methodologies - PDCA Cycle. RADAR Matrix, DMAIC and DFSS Journal of Achievements in Materials and Manufacturing Engineering vol. 43 Issue 1 November 2010.

[2] ANURAG, Bansal, Kuo-Chuan Liu, and Jie Xue. Evaluation of Defects In Lead-Free BGA Solder Joints Under Random Vibration Stress. Cisco Systems, Inc. San Jose, CA, USA .Volume 24 Issue 1, 2011.

[3] FRANCIS, K. H. Lau, Vincent W.S. Yeung. A Hierarchical Evaluation of the Solder Paste Printing Process. Journal of Materials Processing Technology 69 79- 89. 1997.

[4] WHALleY, D.C. A simplified Reflow Soldering Process Model. Journal of Materials Processing Technology 150 134-144. 2004.

[5] KHANDPUR, R. S. Printed Circuit Boards: Design, Fabrication, Assembly and Test. 15rd ed. Chicago: McGraw-Hill, 2006.

[6] BASU, R. Implementing Quality - A Practical Guide to Tools and Techniques. Thomson Learning, London, 2004.

[7] SOKOVIC, M. D Pavletic, Quality improvement - PDCA cycle vs. DMAIC and DFSS. Journal of Mechanical Engineering 53/6 (2007) 369-378.

[8] WEINSTEIN, Jeremy and Vasovski, Steve. The PDCA Continuous Improvement Cycle Module 6.4. Presentation for: ESD.60 - Lean/Six Sigma Systems MIT Leaders for Manufacturing Program (LFM) Summer 2004. 
[9] ASPANDIAR, Raiyo, Mark Litkie, George Arrigotti. Pin in Paste Solder Process Development. Intel Corporation.Conference: SMTA International, 1999.

[10] A SMT- Compatible Technology to include Through Hole Solder Mounted Components into a typical SMR Process: PIP Technology Overview. Available in: www.rcmicro.es/pdf/noticias/FCI_PIN_In_Paste.pdf. Accessed April $11,2015$.

[11] BERNTSON, Ross, B., Ronald Lasky, Ph.D., PE, Karl P. Pfluke. Through-Hole Assembly Options for Mixed Technology Boards. Indium Corporation of America Utica, NY, 2004.

[12] IPCA-610D - Association Connecting Electronics Industries Acceptability of Eletronic Assemblies. February, 2005.

[13] KRASTEV, Evstatin and David Bernard . Modern 2D/3D X-Ray Inspection-Emphasis on BGA, QFN 3D Packages and Counterfeit Components. Conference: Pan Pacific Symposium. Company: Nordson DAGE. 2010.

[14] JAY, Roger \& Alfred Kwong. Dealing with the "black pad defect"- A Failure Analyst's Perspective. Solectron Corporation. SMTA Conference, 2001.

[15] EMPFASIS. National Electronics Manufacturing Center of Excellent. Moisture Sensitive Devices (QFPs and BGAs) 2001. Available http://www.seikausa.com/sites/default/files/product/ mcdry/empf-asis-may-2001.pdf. Accessed April 10, 2015 